\title{
Références bibliographiques du dossier « Les ONG et l'éducation »
}

Hélène Beaucher

\section{(2) OpenEdition}

12 Journals

Édition électronique

URL : http://journals.openedition.org/ries/2214

DOI : 10.4000/ries.2214

ISSN : 2261-4265

Éditeur

Centre international d'études pédagogiques

Édition imprimée

Date de publication : 1 décembre 2011

Pagination : 133-145

ISBN : 978-2-85420-593-0

ISSN : $1254-4590$

\section{Référence électronique}

Hélène Beaucher, "Références bibliographiques du dossier « Les ONG et l'éducation » », Revue internationale d'éducation de Sèvres [En ligne], 58 | décembre 2011, mis en ligne le 01 décembre 2011, consulté le 30 avril 2019. URL : http://journals.openedition.org/ries/2214; DOI : 10.4000/ries.2214

(c) Tous droits réservés 


\section{Références \\ bibliographiques}

\section{Hélène Beaucher}

L'action des organisations non gouvernementales (ONG) en éducation est riche et diverse, mais encore mal connue et la recherche en éducation n'a pas consacré de nombreuses publications à ce sujet. La bibliographie, non exhaustive, s'attache à présenter la diversité des activités, des acteurs et des enjeux de l'implication des ONG dans le domaine de l'éducation. Elle recense des rapports, ouvrages et articles pour l'essentiel publiés depuis moins de dix ans.

La première partie apporte des éléments de cadrage, en traitant du thème des ONG en général, et en présentant, à titre indicatif, quelques cadres politiques et financiers en lien avec l'action des ONG en éducation. Il est impossible de recenser les innombrables plans, nationaux, régionaux et internationaux. La deuxième partie recense des références qui illustrent les principaux domaines d'action des ONG dans le domaine de l'éducation. La troisième partie est consacrée à la question des relations des ONG avec les États, les bailleurs de fonds et la société civile. Quelques études nationales et une courte sitographie complètent cette bibliographie.

Bibliographie arrêtée le 20 octobre 2011.

\section{ÉLÉMENTS DE CADRAGE Qu'est-ce qu'une ONG ?}

\section{DOUCIN Michel, ONG : le contre-pouvoir ?, Editions Toogezer/Paris, 366 p., 2007}

L'auteur analyse les fondements philosophiques et historiques de l'irruption des organisations non gouvernementales $(\mathrm{ONG})$ dans la politique internationale, en décryptant leurs relations avec les États, les organisations internationales, les entreprises et les médias. Cette recherche s'organise en plusieurs étapes : comment les ONG ont-elles émergé dans l'histoire des relations internationales? Leurs rapports d'opposition aux États sont-ils l'élément constitutif essentiel de leur identité? En agrégeant autour d'elles d'autres types d'organisations, quelle forme de pouvoir construisent-elles? En quoi sont-elles une manière nouvelle pour les citoyens de s'impliquer dans la vie politique?

\section{Handicap International (sous la direction de), Contestataires, contestées... Quel avenir pour les ONG dans la nouvelle gouvernance mondiale ? Éditions Dalloz/ Iris/Paris, 2008, 488 p. (Enjeux stratégiques)}

Cet ouvrage réunit les contributions apportées lors du colloque international organisé en 2008 par Handicap International, en partenariat avec l'Institut de relations internationales et stratégiques (IRIS) et Coordination Sud. La première partie traite du thème de la gouvernance mondiale et de la solidarité. Ce thème s'articule autour de deux questions : les ONG sont-elles des acteurs incontournables ou menacés d'une nouvelle marginalisation ? Quels sont les rôles des différents acteurs de la scène internationale? La deuxième partie tente de cerner l'influence des ONG sur les règles de la gouvernance mondiale. Les auteurs abordent ensuite le thème de la place des ONG et de leurs coalitions dans la structuration de l'espace international, en discutant, d'une part, de l'essor de la crédibilité, et de l'influence structurelle des ONG, et d'autre part, du partage des rôles en matière de solidarité entre les ONG et les acteurs privés. Enfin, la dernière partie traite des processus participatifs et de la reconfiguration des rôles et responsabilités des différents acteurs, notamment de la société civile. 
RYFMAN Philippe, Les ONG, La Découverte/Paris, 2009 [2004, première édition], 126 p. (Repères)

Les ONG sont très présentes dans l'actualité mondiale mais finalement assez mal connues. D'autant que, s'il existe une abondante littérature sur les ONG, la recherche demeure encore lacunaire. Cet ouvrage s'attache, après avoir montré l'inscription des ONG dans une histoire déjà longue, à souligner l'ambiguïté du concept. Il s'efforce de répondre aux principales questions les concernant : financement, sociologie, gestion, management, valeurs, identité collective, concurrence, professionnalisation, légitimité, gouvernance, place dans la "société civile » et les relations internationales. Enfin, la montée en puissance des ONG du Sud et des pays émergents reflète une mondialisation qui modifie les contextes d'intervention classiques.

\section{TROUBÉ Christian, L'humanitaire en turbulences. Les ONG face aux défis de} la solidarité internationale, Éditions Autrement/Paris, 2006, (Acteurs de la société) Comment fonctionnent les ONG ? Quelle est leur place dans le nouvel ordre mondial ? Quels sont leurs rapports avec les États, l'Union européenne, les institutions internationales? Comment concilient-elles bénévolat et professionnalisation? À travers trente portraits d'acteurs de terrain et de dirigeants, l'auteur fait le point sur toutes ces questions éthiques, humaines et politiques soulevées par ces organisations.

ZIMET Joseph, Les ONG. De nouveaux acteurs pour changer le monde, Éditions Autrement/Paris, 2006, 80 p. (Monde d'aujourd'hui)

Cet ouvrage propose une vision concrète, globale et synthétique des ONG. Il offre des clés pour comprendre leur nature et leur fonctionnement : leurs liens avec la mondialisation, leurs origines, leur histoire, les différentes familles d'ONG (plaidoyer, urgence, développement, protection de l'environnement), leur organisation interne (les métiers, le financement, la communication). Enfin, il s'interroge sur les critiques auxquelles les ONG doivent faire face, notamment à propos de leur légitimité et de leur transparence.

\section{Cadres politiques publics d'intervention}

Agence française de développement: AFD, département technique et opérationnel, Division éducation et formation professionnelle, Cadre d'intervention sectoriel 2010-2012. Éducation et formation, AFD/Paris, 2010, 54 p.

Le Cadre d'intervention sectoriel (CIS) 2010/2012 pour l'éducation et la formation s'inscrit dans le cadre de la stratégie de coopération française. Il décline la mise en œuvre de la stratégie sectorielle par l'AFD. Il analyse à cet effet les enjeux mondiaux de l'éducation et de la formation, puis les stratégies de la coopération française et internationale dans le secteur, avant de présenter les propositions de soutien de l'AFD concernant les projets de développement dans les pays. Le CIS se décline en trois axes : Objectifs du millénaire pour le développement (généralisation d'une éducation de base de qualité), croissance (amélioration de l'offre de formation professionnelle) et biens publics mondiaux (adaptation au changement climatique).

European Commission, Investing in people. Strategy paper for the thematic programme 2007-2013, OPOCE /Luxembourg, 2011, 21 p.

Guidé par l'ambition d'aider les pays partenaires de la Communauté européenne à atteindre les Objectifs du millénaire pour le développement, ce programme thématique soutient des activités relevant de quatre axes : la santé pour tous ; l'éducation, la connaissance et les compétences; l'égalité des sexes; l'emploi et la cohésion sociale. Dans le domaine de l'éducation, la principale priorité concerne la promotion d'une instruction primaire de qualité pour tous par 
la stimulation de l'augmentation du nombre d'inscriptions et par l'encouragement de l'égalité d'accès à une instruction primaire de qualité. Le programme vise également la création des conditions nécessaires à l'amélioration de l'accès à la formation professionnelle et au renforcement des compétences.

\section{$I A D B$ : Inter-American Development Bank, Sector guidelines for education, IADB/Washington, 2011, 34 p.}

Dans les prochaines années, pour les pays dans lesquels la Banque interaméricaine de développement intervient, il s'agit, d'une part de réduire la pauvreté et les inégalités, d'autre part de promouvoir une croissance durable. Ces lignes directrices se déclinent en cinq objectifs stratégiques dans le secteur de l'éducation : l'amélioration des services éducatifs pour le développement de la petite enfance ; l'amélioration de la formation des enseignants et des environnements d'apprentissage ; la facilitation de la transition école/travail ; le soutien au développement des programmes; la promotion de l'évaluation de la qualité de l'apprentissage.

\section{Nations Unies, Objectifs du millénaire pour le développement. Rapport de 2011,} Nations Unies/New York, 2011, 72 p.

Adoptée lors du sommet des Objectifs du millénaire pour le développement (OMD) en 2000 par les dirigeants du monde entier, la Déclaration du millénaire a fixé huit objectifs pour faire progresser le développement et réduire la pauvreté d'ici 2015 : réduire de moitié la pauvreté et la faim ; réaliser l'éducation primaire universelle ; réaliser l'égalité des sexes ; réduire de deux tiers la mortalité infantile : réduire de trois quarts la mortalité maternelle ; inverser la tendance en matière de propagation du VIH-Sida et du paludisme; réaliser un développement durable et assurer la viabilité de l'environnement; mettre en place un partenariat mondial pour le développement. Ce rapport annuel présente les évaluations des progrès réalisés jusqu’à présent.

UNESCO : Organisation des Nations-Unies pour l'éducation la science et la culture, Rapport mondial de suivi sur l'éducation pour tous 2011 - La crise cachée : les conflits armés et l'éducation, UNESCO/Paris, 2011, 463 p.

En 2000, les participants au Forum mondial de l'éducation - enseignants, ministres représentants d'ONG et d'organisations nationales - ont adopté le Cadre d'action de Dakar, l'éducation pour tous (EPT), et fixé six objectifs à atteindre d'ici 2015 : développer et améliorer sous tous leurs aspects l'éducation et la protection de la petite enfance; garantir l'accès de tous les enfants à un enseignement primaire et gratuit de qualité ; améliorer les possibilités d'apprentissage ouvertes aux jeunes et aux adultes; améliorer de $50 \%$ les taux d'alphabétisation des adultes; réaliser l'égalité entre les sexes dans l'éducation; améliorer la qualité de l'éducation et la protection de la petite enfance. Ce rapport évalue les progrès des pays et des régions vers chacun des objectifs. Chaque rapport annuel est consacré à un thème particulier. Le rapport 2011 analyse les conséquences des conflits armés sur l'éducation et propose un programme de réformes et la révision des priorités de l'aide internationale.

\section{World Bank, World Bank group education strategy 2020. Learning for all. Investing in people's knowledge and skills to promote development, The World Bank/Whashington, 2011, 112 p.}

Ce document établit la stratégie d'éducation de la Banque mondiale à l'horizon 2020. «L'apprentissage pour tous " place l'apprentissage au cour de sa stratégie, en insistant sur trois points : investir précocement, investir intelligemment et investir pour tous. Le développement, et la réduction de la pauvreté dépendent des connaissances et des compétences que les personnes doivent acquérir en adéquation avec le marché du travail. La Banque mondiale privilégiera la réforme des systèmes éducatifs nationaux dans leur ensemble, afin d'améliorer la qualité de l'apprentissage des élèves. Elle calibrera les nouveaux financements alloués à l'éducation en fonction des résultats. Enfin, elle élaborera une base de connaissances pour la réforme de l'éducation. 


\section{DE L'ACTION OPÉRATIONNELLE \\ À LA CONTRIBUTION \\ AUX POLITIQUES PUBLIQUES}

\section{Actions de plaidoyer}

AKMAN Sheila, "Marching to different rhythms: international NGO collaboration with the state in Tanzania ", Development in practice, vol. 20, $n{ }^{\circ} 4-5$, p. 498-510

Cette contribution présente les différentes stratégies mises en place par OXFAM GB en Tanzanie. Elle analyse ce que signifie une "approche programme " pour les travaux menés par cette ONG dans le secteur de l'éducation et se penche plus particulièrement sur ses partenariats et relations de plaidoyer aux niveaux local et national avec différents agents et institutions publics du secteur de l'éducation. Le document conclut que les pratiques de plaidoyer doivent être considérées comme multiples, s'inscrivant dans des processus.

DOLAN Janice, NDARUHUTSE Susy, Save the Children UK's financial flows, programme choices, and the influences of the Rewrite the Future Campaign, 2010, 39 p. disponibles sur le site: http://unesdoc.unesco.org [en ligne]

Ces dernières années, l'ONG Save the Children a fait de l'éducation des enfants touchés par les conflits une priorité à travers sa campagne « Réécrire le futur ». En augmentant significativement les ressources allouées aux programmes dans les pays touchés par les conflits, l'organisation a augmenté ses programmes d'éducation dans ces contextes. La campagne a également eu un impact international en plaidant pour l'augmentation des flux d'aide aux pays touchés par les conflits. Elle a sensibilisé la communauté internationale à la nécessité de donner accès à l'éducation aux enfants touchés par les conflits.

\section{Éducation formelle et non formelle}

CASELY-HAYFORD Leslie, HARTWELL Ash, "Reaching the underserved with complementary education: lessons from Ghana's state and non-state sectors ", Development in Practice, vol. 2, $n^{\circ} 4-5$, juin 2010, p. 527-539

Entre 1995 et 2006, plus de 85000 enfants âgés de 8 à 14 ans ont participé à un programme d'éducation complémentaire dans des zones rurales du nord du Ghana. School for Life, une organisation à but non lucratif, propose neuf mois d'instruction dans la langue parlée par les enfants. Une évaluation de l'impact du programme a démontré que les programmes d'éducation complémentaire sont en mesure d'aider les enfants à atteindre un niveau d'alphabétisme de base dans leur langue maternelle dans un délai plus bref et de manière plus économique que ne le peuvent les systèmes de scolarité primaire de l'État.

\section{COMPAORE Maxime, Évaluation du programme éducation en milieu pastoral} dans le Sahel du Burkina Faso. Evaluation report, Oxfam GB/Oxford, 2008, 39 p.

Cette étude vise à évaluer le plan d'action de trois ans initié par Oxfam et exécuté au Burkina Faso par une ONG locale, l'association Nodde Nooto (A2N). Le programme comportait cinq composantes : accès et rétention à l'école, qualité de l'éducation, promotion de l'équité et de l'égalité des sexes, renforcement des capacités de la société civile, et appui aux acteurs locaux. L'engouement que connaît l'école dans les zones d'intervention du projet fonde l'auteur à dire que les activités menées ont correspondu aux besoins réels des bénéficiaires. 
OCHSE Katharina, Fit for life? Non-formal post-primary initiatives in Yemen, Malawi and Naminbia, GTZ: Deutsche Gesellschaft für Technische Zusammenarbeit/ Eschborn, 2008, 72 p.

Un nombre croissant d'élèves d'Afrique subsaharienne frappent aux portes des établissements d'enseignement secondaire sans y être admis ; lorsqu'ils le sont, le taux d'abandon des études est très élevé. Cet ouvrage analyse l'état de l'éducation post-primaire en Afrique subsaharienne et en particulier au Yémen, en Namibie et au Malawi. Il illustre l'importance des ONG dans le domaine des initiatives post-primaires non formelles.

\section{Enseignement et formation techniques et professionnelle (EFTP)}

Fondation Paul Guérin Lajoie, Le projet d'appui à la formation professionnelle des néo-alphabétisés (PAFPNA) au Sénégal : un projet d'alphabétisation efficace prometteur. Document de travail, ADEA : Association pour le développement de l'éducation en Afrique/Paris, 2006, 22 p. disponibles sur le site http:// adeanet.org [en ligne]

Ce document présente le projet PAFPA, mené au Sénégal par une ONG canadienne, la fondation Paul Guérin Lajoie, à l'issue de deux années de mise en œuvre. Le projet porte sur l'apprentissage de métiers prometteurs d'emploi et adaptés aux jeunes marginalisés par le système formel de formation. Il entend contribuer au développement de la formation professionnelle, en accompagnant la mise en place d'un modèle de formation qui prend appui sur l'apprentissage en milieu non formel dispensé dans les ateliers de maîtres artisans. 350 apprentis ont bénéficié de cette formation pratique d'une durée totale de quatorze mois. Malgré des résultats positifs, la question de la pérennisation du projet se pose.

\section{Éducation pour les populations rurales}

Unité de coordination du partenariat éducation pour la population rurale (EPR), Les ONG françaises et le programme FAO/UNESCO "Éducation pour les populations rurales" (EPR), FAO : Organisation des Nations unies pour l'alimentation et l'agriculture/Rome, 2005, 76 p. disponibles sur le site : http://www.fao.org [en ligne]

Le programme EPR vise à donner accès à l'éducation de base aux populations rurales en renforçant le partenariat entre tous les acteurs de l'éducation. Il s'inscrit dans une double logique de lutte contre la pauvreté et de sécurité alimentaire. Sous la forme de fichesprojets, ce document constitue un panorama commenté des initiatives entreprises par vingt-sept ONG françaises en matière d'éducation formelle et non formelle. Formation professionnelle agricole, alphabétisation, vulgarisation technique, mises en places de centres de formation technique, éducation à la santé, appui aux associations de parents d'élèves, centre de formation, formation technique, prêts, etc.: ces initiatives sont extrêmement diversifiées. 


\title{
Renforcement des capacités
}

NEU Daniel, GANDZOUNOU Reiye, Évaluation intermédiaire du programme concerté de renforcement des capacités de la société civile et de la jeunesse guinéenne (PROJEG). Rapport final, F3E/Paris, juillet 2010, 71 p.

En Guinée, le DSRP (Document stratégique de réduction de la pauvreté qui fixe les priorités de l'action publique) recommande la participation de la société civile à l'élaboration des politiques publiques. Ce rapport rend compte de l'évaluation intermédiaire du PROJEG, dont le chef de file est l'ONG française Aide et action. PROJEG a pour objectif de faciliter, dans le cadre d'un partenariat pluri-acteurs, la participation de la société civile et de la jeunesse guinéenne à la formulation et à la mise en œuvre des politiques publiques sectorielles de développement. L'obligation d'agir en consortium, telle que formulée dans les termes de références, constitue une innovation.

\section{RELATIONS DES ONG AVEC LES ÉTATS, LES BAILLEURS DE FONDS ET LA SOCIÉTÉ CIVILE}

\begin{abstract}
MILLER-GRANDVAUX Yolande, WELMOND Michel, WOLF Joy, Evolving Partnerships. The Role of NGOs in Basic Education in Africa, United States Agency for International Development/ 2002, 82 p. disponibles sur le site http:// pdf.usaid.gov [en ligne]

Les auteurs étudient le rôle que les ONG jouent dans le secteur de l'éducation en Afrique. Ils analysent l'évolution des relations des ONG avec les gouvernements et les bailleurs de fonds, les implications des interventions des ONG au niveau des systèmes éducatifs et de la société civile. Ils examinent enfin les facteurs contextuels ayant influencé les programmes des ONG. L'étude circonscrit quatre axes principaux : les relations entre les ONG et le gouvernement, l'influence des ONG sur les politiques éducatives, la relation entre les ONG et les bailleurs de fonds et l'influence des ONG sur la société civile.
\end{abstract}

\section{Les ONG et les États}

BATLEY Richard, ROSE Pauline, "Collaboration in delivering education: relations between governments and NGOs in South Asia ", Development in practice, vol. $20, \mathrm{n}^{\circ} 4-5,2010$, p. 579-585

La collaboration entre les gouvernements et les prestataires non publics de services de base suscite une attention croissante de la part des agences internationales et des décideurs nationaux. Elle vise à soutenir des buts communs pour parvenir à une prestation de services universelle. En se basant sur des recherches menées au Bangladesh, en Inde et au Pakistan, cet article montre que la collaboration peut porter ses fruits lorsque les ONG ne dépendent pas de sources limitées pour leur financement, et investissent du temps dans la construction d'une relation informelle avec les gouvernements. Non seulement la collaboration peut renforcer la prestation de services par les ONG, mais elle donne également l'occasion à celles-ci de s'engager dans des activités de plaidoyer plus larges. 
JAGANNATHAN Shanti, The role of nongovernmental organizations in primary education. A study of six NGOs in India, World Bank Policy Research Working Paper $n^{\circ}$ 2530, 2000, 62 p.

En Inde, des ONG permettent aux enfants défavorisés d'accéder à l'éducation et développent des innovations pédagogiques qui améliorent la qualité de l'enseignement primaire. Dans cette étude qui porte sur six ONG œuvrant pour l'éducation élémentaire, l'auteur montre les avantages potentiels d'une alliance gouvernement-ONG pour atteindre l'éducation primaire universelle. La collaboration peut se révéler particulièrement fructueuse pour cibler les enfants les plus démunis et améliorer la qualité de l'éducation, notamment grâce à la formation des enseignants à ce public.

NISHIMUKO Mikako, "The role of non-governmental organisations and faithbased organisations in achieving education for all: the case of Sierra Leone ", Compare: a journal of comparative and international education, vol. $39, n^{\circ} 2$, mars 2009, p. 281-295

La Sierra Leone, l'un des pays les plus pauvres du monde, a connu une guerre civile de 1991 à 2002. La capacité du gouvernement à fournir des services éducatifs demeure faible et $30 \%$ des enfants n'ont pas accès à l'enseignement primaire. Ce document traite du rôle des ONG et des organisations confessionnelles dans ce domaine, et notamment de leur appui au gouvernement. L'auteur conclut que le travail mené en collaboration entre le gouvernement, les ONG et les organisations confessionnelles a permis des progrès vers la réalisation de l'éducation pour tous.

ULLEBERG Inger, The role and impact of NGOs in capacity developpement. From replacing the state to reinvigoring education. Rethinking capacity development, IIEP/Paris, 2009, 51 p. disponibles sur le site http://www.iiep.unesco.org [en ligne]

Selon l'auteur, les ONG sont devenues les principaux acteurs du service public dans les pays où l'État n'est pas en mesure de fournir les services nécessaires. Puisque le discours international de développement s'oriente de plus en plus vers le développement de compétences et d'outils pour renforcer la société, les ONG s'adaptent. Cette étude examine le rôle des ONG dans le développement international à travers le développement des capacités, domaine où les ONG sont de plus en plus impliquées. Les activités de développement des capacités complètent les fonctions traditionnelles des ONG. La nouvelle interprétation du développement des capacités peut affaiblir l'État central, mais peut également le renforcer sur le long terme. Les ONG peuvent innover et s'adapter plus rapidement qu'un gouvernement national ; elles peuvent parfois nuire aux efforts de l'État. Si les ONG ont un impact non négligeable sur le processus de développement des capacités, elles continuent de souffrir du manque de ressources et de l'éloignement de l'État. En tous les cas, les activités des ONG sont de plus en plus diverses.

\title{
Les ONG et les bailleurs de fonds
}

\begin{abstract}
ActionAid International, Care International. Where to now? Implications of changing relations between DFID, recipient governments and NGOs in Malawi, Tanzania and Uganda, ActionAid International/London, 2006, 82 p. disponibles sur le site: http://www.careinternational.org [en ligne]

Le contexte de l'aide au développement a beaucoup changé depuis 1996, avec, du côté des bailleurs, l'apparition de la notion de responsabilité financière, de l'appui budgétaire direct, et la promotion des partenariats et de la participation de la société civile à la formulation des politiques. Ce rapport rassemble des recherches menées au Malawi, en Tanzanie et en Ouganda.
\end{abstract}


Il explore les conséquences politiques et financières, pour les ONG, des changements majeurs survenus dans les politiques des bailleurs de fonds, notamment l'aide gouvernementale britannique (DFID : Departement for International Development) depuis la fin du siècle dernier. Si le passage à une aide financière directe n'affecte pas particulièrement le niveau de financement des ONG, il entraîne des changements dans le type d'activités financées.

\section{World Bank, Issues and options for improving engagement between the world bank and civil society organizations, World Bank/Washington, 2005, 75 p.}

La collaboration avec la société civile, notamment la prise en compte des desiderata des populations démunies, la participation des citoyens à la définition des politiques gouvernementales contribuent, selon la Banque mondiale, à l'amélioration des programmes, et accélèrent les progrès vers la réalisation des Objectifs du millénaire. Ce rapport vise à évaluer les relations récentes de la Banque mondiale avec les organisations de la société civile (OSC), société civile désignant un «large éventail d'organisations non gouvernementales qui ont une place certaine dans la vie publique, véhiculent les préoccupations et les valeurs de leurs membres ou autres sympathisants, sur la base de considérations d'ordre éthique, culturel, scientifique, religieux ou philanthropique ». Les auteurs analysent les mutations survenues depuis l'examen de 1998 intitulé The Bank's Relations with NGOs: Issues and Directions, et recommandent une participation plus effective de la société civile aux actions soutenues par la Banque.

\section{Vers l'émergence de la société civile?}

KLEES Steven J., "NGOs, civil society, and development: is there a third way? ", Current Issues in Comparative Education, vol. 10, $n^{\circ} 1-2,2008$, p. 22-25

La plupart des débats sur la question d'une «troisième voie " pour le développement se sont concentrés sur les rôles que peuvent jouer les ONG et les autres acteurs de la société civile. L'auteur examine les façons dont les forces sociales et mondiales compromettent la capacité des ONG à réaliser des réformes durables. Il propose un nouveau modèle de réforme qui intégrerait, dans une organisation véritablement transformationnelle, activisme citoyen, démocratie participative, justice sociale et mise en réseau.

MUNDY Karen, "From NGOs to CSOs: social citizenship, civil society and "Education for all" - an agenda for further research ", Current Issues in Comparative Education, vol. 10, $n^{\circ} 1-2,2008$, p. 32-40

Il y a dix ans, les discussions portaient sur le rôle croissant des ONG dans le domaine de l'éducation. Aujourd'hui, le discours a changé, et aussi, à certains égards, les pratiques. Dans cet article, l'auteur examine le nouveau visage de la société civile à la lumière des discours de l'aide au développement, et notamment en tentant de répondre à la question du rôle que joue la société civile dans la réalisation de l'EPT. L'article examine comment le concept d'engagement de la société civile s'est construit dans le discours officiel et politique tenu par les ONG de l'aide au développement, et il en fait ressortir ses limites.

MUNDY Karen, HAGGERTY Megan, SIVABRAMANIAM Malini Cherry, "Civil society, basic education, and sector wide aid: insights from Sub-Saharan Africa ", Development in practice, vol. $20, n^{\circ} 4-5$, p. 484-497

Les tendances émergentes des réformes des plans du secteur de l'éducation indiquent une évolution non seulement de la manière dont l'aide étrangère est versée mais aussi de la façon dont les acteurs de la société civile assument de nouveaux rôles ayant trait aux politiques générales et au plaidoyer. Cette contribution examine les rôles en mutation de la société civile 
dans quatre pays : le Burkina Faso, le Kenya, le Mali et la Tanzanie. Si les approches sectorielles ont effectivement créé de nouvelles occasions de participation de la société civile au niveau national, les auteurs suggèrent que les réformes du secteur ont également soulevé des défis considérables pour le dialogue avec les pouvoirs publics et les bailleurs de fonds. Elles mettent l'accent sur la nécessité d'un processus transparent, réglementé et démocratique pour l'inclusion des organisations de la société civile dans les débats portant sur les politiques.

\section{PETIT Pierre (sous la direction de), Société civile et éducation; le partenariat} à l'épreuve du terrain, Bruylant-Academia/Louvain-la-Neuve, 2010, 224 p.

Dans le discours mondialisé du développement, la mobilisation de tous les acteurs concernés a été prescrite, au premier rang desquels les organisations de la société civile (OSC), censées apporter de nouvelles ressources et instaurer progressivement une «bonne gouvernance » impliquant des espaces participatifs à tous les niveaux. Les auteurs entendent ouvrir une perspective critique qui remette en cause " une approche consensualiste reflétant avant tout des choix de société élaborés au sein d'institutions internationales ». Cet ouvrage offre des études empiriques réalisées au Bénin, au Congo, au Sénégal et en Argentine, sur l'action des OSC actives en matière d'éducation dans les pays du Sud. Une série d'hybridations apparaît, ainsi que des divergences entre la rhétorique de la gestion participative et les logiques pratiques à l'œuvre sur le terrain.

\section{Quelques Études de CAS}

\section{Afrique}

\section{$B A R R Y$ Billo, Le rôle des ONG dans l'éducation de base en Guinée, United States} Agency for International Development: USAID/Conakry, janvier 2002, 29 p.

Cette étude décrit les domaines d'intervention ainsi que les types d'ONG opérant dans le secteur de l'éducation en Guinée. L'auteur analyse l'impact de l'action des ONG dans la mise en ouvre de la politique éducative, avant de tirer les leçons qui permettent d'apprécier les acquis et de mettre en relief les enjeux réels des ONG dans le secteur.

\section{COMHAIRE Gaël, STANGHERLIN Gregor, Les organisations de la société civile} dans le secteur de l'éducation de base au Bénin. Rapport de la mission du 29 mai au 6 juin 2005, GRAP-OSC: Groupe de recherche en appui à la politique aux organisations de la société Civile/Liège, 2005, 64 p.

Qui sont les acteurs impliqués dans le système éducatif béninois ? Quels rôles jouent les organisations de la société civile (OSC) dans ce système? Que développent-elles comme projet éducatif ? Quelles relations entretiennent-elles avec les pouvoirs publics locaux et nationaux, avec les partenaires internationaux? Telles sont les principales questions autour desquelles s'organise ce rapport. La mission avait pour objectifs de réaliser un inventaire des différentes initiatives dans l'éducation de base, de clarifier les différentes fonctions des OSC (prestataire de services, mobilisation des communautés, agents d'innovation, plaidoyer), ainsi que d'étudier les rapports des OSC avec l'État, les bailleurs de fonds et les OSC du Sud.

KADZAMIRA Esme Chipo, KUNJE Demis, The changing roles of nongovernmental organizations in education in Malawi, United States Agency for International Development (USAID), Bureau for Africa, Centre for Educational Research and Training of the University of Malawi/Zomba, mars 2002, 47 p. disponibles sur le site: http://pdf.usaid.gov [en ligne]

Les auteurs retracent l'histoire de l'implication des ONG dans le domaine de l'éducation au Malawi. La nature des programmes mis en œuvre semble déterminer à la fois les relations entre les ONG et le gouvernement et les relations entre les ONG et l'État. Si les ONG 
parviennent à infléchir certaines politiques éducatives, elles ne les changent pas. Les opérations des ONG devraient être réglementées, le manque de crédibilité et de transparence ayant contribué à la réticence des bailleurs de fonds et de l'État à financer les activités des ONG.

\section{LAMARKBI Nadia, LAFAYE DE MICHEAUX Elsa, "L'ouverture de l'éducation} primaire rurale aux ONG. Les enseignements du Maroc contemporain », Mondes en développement, $n^{\circ} 134,2006, p$. 79-94

Dans un contexte d'analphabétisme et de sous-scolarisation des enfants de paysans, le gouvernement marocain a fait officiellement appel aux ONG en 1998, sous la pression des organisations internationales, pour assurer la formation des élèves de zones rurales isolées. À partir d'une enquête de terrain sur la Fondation Zakoura, cet article étudie l'émergence d'un nouveau marché scolaire et tente de cerner les contours de l'enseignement reçu par ces enfants. Il apparaît que le contenu et le niveau académique de l'enseignement offert n'est pas à la hauteur du système éducatif national, malgré l'annonce d'innovations pédagogiques.

TOUNKARA Bréhima, Le rôle des ONG dans l'éducation de base au Mali, USAID : United States Agency for International Development, ROCARE : Réseau Ouest et Centre Africain de Recherche en Education, ROCARE/Bamako, décembre 2001, 40 p.

Au Mali, souvent cité en exemple pour l'implication des ONG dans le développement de l'éducation, le rôle des ONG est diversement apprécié. La nature des relations entre les bailleurs de fonds, les représentants de l'État et les ONG fluctue selon l'envergure nationale ou internationale de ces dernières. La problématique de l'intervention des ONG dans le secteur de l'éducation, essentiellement axée sur le développement des écoles communautaires, aboutit à un certain nombre de constats souvent controversés. Les ONG sont en mesure de s'implanter dans le secteur quand une politique de tolérance est mise en œuvre tôt. L'auteur préconise de légiférer et de définir les cadres et les modalités d'intervention dans le développement de l'éducation.

\section{Amérique latine}

\section{ADELMAN Elizabeth, SCHUH MOORE Audrey-Marie, JAMOVICH Analia,} Using Opportunity to learn and early grade reading fluency to measure school effectiveness in Honduras, USAID/Washington, 2010, 32 p.

Cette étude se situe dans le cadre du programme d'amélioration de la qualité de l'éducation «EQUIP2 ». Elle évalue l'efficience des écoles mises en place par l'ONG Care. Les auteurs ont cherché à déterminer si ces écoles offraient la possibilité d'apprendre à lire couramment dans la langue d'enseignement, l'espagnol. Ils se sont également penchés sur l'utilisation de la journée scolaire, ponctuée par de longues pauses. Il ressort de cette étude que les élèves des écoles Care ont des compétences en lecture supérieures à celles des élèves des autres écoles, en dépit de certaines faiblesses dans le domaine de la compréhension des textes.

\section{CARE France, Qualité et équité de l'éducation biculturelle du département de Puno, Pérou, CARE France/Paris, 2011, 14 p.}

Ce document présente un projet de l'ONG Care au Pérou. Il est financé à $75 \%$ par la Commission européenne, Care Pérou et Care France apportant le financement restant. Ce projet cherche à repenser l'éducation dans le cadre de l'éducation interculturelle bilingue, en proposant un programme éducatif qui promeut une formation permettant aux bénéficiaires d'acquérir les aptitudes nécessaires pour affronter les problèmes, être capables d'auto-apprendre et de s'adapter à des connaissances changeantes. Il s'agit d'améliorer la qualité de l'apprentissage des garçons et des filles inscrits dans les établissements interculturels bilingues, ainsi que de renforcer les capacités de gestion pédagogique des fonctionnaires régionaux et locaux, éducateurs et instituteurs. 


\section{Asie}

BANO Masooda, "Non-profit education providers vis-à-vis the private sector: comparative analysis of non-governmental organizations and traditional voluntary organizations in Pakistan ", Compare: a journal of comparative and international education, vol. $38, n^{\circ} 4,2008$, p. 471-482

Les institutions internationales de développement ont largement fait la promotion des ONG dans les pays en développement. À partir d'une enquête menée au Pakistan, ce document montre que le secteur de ces prestataires n'est pas monolithique. En effet, les ONG ouvrant dans le secteur de l'éducation sous le patronage de donateurs internationaux suivent des modèles de prestation nettement différents des modèles utilisés par les organisations bénévoles traditionnelles, qui dépendent des dons nationaux. Si la philosophie du service social des organismes traditionnels les distingue nettement du secteur privé, ce n'est pas le cas des autres ONG. L'auteur soutient que ces ONG, plutôt que de satisfaire les besoins éducatifs des plus pauvres, opèrent selon un modèle de prestation qui finit par alimenter des marchés au profit du secteur privé.

BLUM Nicole, "Small NGO Schools in India: implications for access and innovation ", Compare: a journal of comparative and international education, vol. $39, n^{\circ} 2$, mars 2009, p. $235-248$

Si les écoles privées proliferrent en Inde, les ONG jouent également un rôle important dans le domaine de l'enseignement primaire, notamment auprès des communautés défavorisées. Ce document interroge le potentiel des ONG; il explore en particulier les contributions d'un de leurs programmes, qui a cherché à accroître l'accès des enfants marginalisés à l'éducation, en fournissant un appui aux petites écoles rurales. L'auteur soutient que les programmes des ONG de ce type ont des impacts positifs en termes d'accès et de qualité de l'éducation. En effet, les programmes sont menés à petite échelle et enracinés localement. Flexibles, les ONG sont dotées d'une plus grande capacité d'innovation que l'État dans les domaines tels que la conception des curricula et la formation des enseignants.

IQBAL Javed, "Role of non-governmental organizations for the development of basic education in Punjab, Pakistan ", Educational research and review, vol. 5, $n^{\circ}$ 8, août 2010, p. 423-426

L'objectif de l'article est d'étudier le travail de cent douze ONG œuvrant pour l'éducation de base et l'alphabétisation au Pendjab, ainsi que leurs services et d'équipements éducatifs. L'enquête a été menée auprès d'un échantillon de responsables d'ONG, d'enseignants, de parents d'élèves et d'experts du secteur éducatif. Elle recommande que la municipalité ou le gouvernement subviennent aux besoins des établissements gérés par les ONG et dont les locaux et l'équipement sont inadaptés.

\section{France}

BARLET Sandra, BARON Christian, LAGANDRE Damien, DELLUC André, Regards sur la coopération française en matière d'éducation et de formation professionnelle, GRET/Paris, 2008, 106 p. (Études et Travaux en ligne) disponibles sur le site http://www.gret.org [en ligne]

Ce document propose un état des lieux des actions de coopération française en matière d'éducation, de formation professionnelle et d'insertion. Il situe la coopération publique française dans son contexte international, et présente la stratégie suivie par la France. Une enquête menée auprès de plus de soixante ONG, et complétée par des entretiens individuels avec quelques fondations et collectivités, permet de tracer les grandes lignes du paysage de la coopération française non gouvernementale. 


\title{
QUELQUES SITES
}

\section{Sites d'ONG}

\begin{abstract}
Aide et Action
Aide et action est l'une des principales ONG françaises spécialisées dans l'éducation. En 2009, Aide et Action a mis en œuvre plus de cent projets dans vingt-deux pays. Ses activités se déploient dans le cadre de six axes : favoriser l'accès à l'éducation et améliorer les conditions d'accueil des enfants, améliorer la qualité de l'éducation, appuyer les communautés dans le développement de leur projet éducatif, favoriser l'intégration sociale et professionnelle des plus vulnérables, sensibiliser et mobiliser l'opinion publique mondiale pour un monde plus juste et équitable, et garantir l'éducation dans l'urgence et la post-urgence. http://www.aide-et-action.org/.
\end{abstract}

\section{ATD Quart Monde}

Présente dans une trentaine de pays dans le monde, l'ONG ATD-Quart Monde agit dans les domaines suivants : rejoindre les personnes en situation d'extrême pauvreté, promouvoir l'accès au savoir, à la culture et à l'éducation, mener des projets pilotes pour garantir l'accès de tous aux droits fondamentaux, informer, mobiliser les citoyens, la société civile, les autorités et les institutions, mener une recherche et développer une connaissance, et promouvoir la participation et la représentation des populations très défavorisées vis-à-vis des organismes locaux, nationaux et internationaux. http://www.atd-quartmonde.org/.

\section{Brac: Bangladesh rehabilitation advancement committee}

144 Créée en 1972, au Bangladesh, Brac est une ONG mondiale multiservices gérant divers programmes de développement rural et urbain : programme de santé, d'éducation, et de développement économique. www.brac.net.

\section{Care}

Réseau international de quatorze membres implantés dans quinze pays, Care est l'une des principales ONG américaines, active dans les champs de l'éducation, de la santé, du développement rural, accès à l'eau, développement économique) et dans le domaine de l'urgence. http://www.care.org/.

\section{Gret}

Le Gret est l'une des principales ONG de développement française, active dans une quarantaine de pays. Outre ses actions de développement sur le terrain, le Gret produit et publie des travaux de recherche sur le développement. Le Gret est notamment actif en matière d'appui à la petite entreprise, de formation et d'insertion professionnelles. http://www.gret.org/.

\section{Plan}

Plan est une ONG centrée sur l'enfant et la promotion de ses droits. La santé, l'éducation, la protection de l'enfant, les activités génératrices de revenus, l'environnement, l'eau et l'assainissement sont les domaines d'action prioritaires de l'ONG Plan. Dans le domaine de l'éducation, l'ONG s'investit dans la construction d'écoles, la sensibilisation des familles, le soutien scolaire, les centres d'éducation spécialisés pour les enfants travailleurs, l'amélioration de la qualité de l'éducation en formant des enseignants et en soutenant des projets pédagogiques innovants centrés sur la participation des élèves. Elle promeut l'enregistrement des naissances pour que les enfants puissent disposer de leurs droits et notamment, être inscrits à l'école à l'école. Elle intervient dans cinquante pays en Afrique, en Amérique latine, en Asie. http://www.planfrance.org/. 


\title{
Oxfam
}

Oxfam est une ONG britannique qui a suscité la création d'une confédération internationale de quinze organisations travaillant ensemble dans 98 pays. Elle conduit des actions de développement, d'urgence et de plaidoyer international dans le monde entier. http://www.oxfam.org/.

\section{World vision}

ONG américaine chrétienne multinationale basée dans près de cent pays, World vision agit à travers des programmes de développement (parrainage d'enfants), d'aide humanitaire d'urgence et ses campagnes de plaidoyer. http://www.wvi.org/.

\section{Les plates-formes d'ONG}

\author{
Abong (Brésil) : www.abong.org.br \\ Accion (Chili) : www.accion.org
}

\section{Concord}

L'objectif principal de la confédération des ONG européennes d'urgence et de développement est de renforcer l'impact des ONG européennes auprès des institutions européennes, en combinant expertise et représentation. http://www.concordeurope.org

\section{Congad (Sénégal) : www.congad.sn}

\section{Coordination Sud}

Coordination Sud (Solidarité, Urgence, Développement) est la coordination nationale des ONG françaises de solidarité internationale. Elle rassemble plus de centre trente ONG qui mènent des actions humanitaires d'urgence, d'aide au développement, de protection de l'environnement, de défense des droits humains auprès des populations défavorisées mais aussi des actions d'éducation à la solidarité internationale et de plaidoyer. Coordination Sud assure une double mission d'appui à la professionnalisation des ONG françaises et de représentation de leurs positions auprès des institutions publiques et privées, en France, en Europe et dans le monde. Les ONG membres de Coordination Sud engagées dans le domaine de l'éducation et de la formation se rassemblent dans la commission Éducation et formation professionnelle. Elles veillent notamment à la bonne harmonisation des différentes initiatives prises en France dans le cadre de la campagne mondiale sur l'éducation. http://www.coordinationsud.org/.

\section{Fip : Forum international des plates-formes nationales d'ONG}

Le Forum international des plates-formes nationales d'ONG (FIP) a été créé en 2008 par 82 plates-formes nationales d'ONG et sept coalitions régionales. En 2010, le FIP a contribué à renforcer les plates-formes nationales d'ONG et les coalitions régionales, à faciliter les échanges entre ses membres, notamment à travers des « exercices de diplomatie non gouvernementale (DNG) ", mais aussi via son site Internet, outil quadrilingue d'information, de communication, de coopération et de plaidoyer. http://www.ong-ngo.org/.

Vani (Inde) : www.vaniindia.org

Piango (Îles du Pacifique) : www.piango.org

InterAction (États-Unis) : www.interaction.org/

Espace Associatif (Maroc) : www.espace-associatif.ma

CNONGD (République démocratique du Congo) : www.ong-ngo.org

SADC-Council of NGOs (Afrique australe) : www.sadccngo.org 
